\title{
A Three-Component Model for Change Planning through Employees' Accountability
}

\author{
Abu-Hussain Jamal ${ }^{1}$, Oleg Tilchin ${ }^{2}$ \\ ${ }^{1,2}$ Al-Qasemi Academic College of Education, P.O. Box 124, Baqa El-Gharbieh, 30100, Israel \\ Correspondence: Oleg Tilchin, Computer Science Department, Al-Qasemi Academic College of Education, E- \\ mail:oletilchin@gmail.com
}

\begin{abstract}
The suggested model shapes change planning based on the employees' accountability. Change planning is accompanied by building accountability and determining the quantitative measures of accountability. It is resulted by the peers and management assessments of the employees' change proposals and readiness to participate in realization of peer proposals. The change planning is performed by consistent performance three model components. The components are Create, Assess, and Determine. In Create component managers determine goals and directions of change, form requirements and conditions for qualitative and motivated creation of change proposals, and familiarize employees with goals, directions, requirements and conditions. Then, the employees create change proposals and disseminate their proposals to the co-workers. In Assess component the managers and employees assess the presented proposals according to the types, requirements, and conditions of assessment. Along with that, readiness including interest, opportunity, and ability, to participate in realization of the proposed changes is also assessed. It allows building collaborative teams and assessing the employees' involvement in change process. In Determine component the measure of the team' mutual accountability for the change proposal realization is determined and it is divided among team members on the basis of quantitative assessment of personal readiness to participate in the change. Then, change planning guided by the accountability measures are performed. The most accountable team leader, the most accountability significant proposal in the change direction, and the most accountability significant direction are identified. The employees' compensation for participation in the change planning adapted to measures of the employees' accountability for realization change is calculated.
\end{abstract}

Key words: change planning, accountability for change

DOI: $10.7176 / \mathrm{EJBM} / 12-12-14$

Publication date: April $30^{\text {th }} 2020$

\section{Introduction}

The need for effective performance of organizational change encourages managers to determine the actual goals and associated with them change directions, engage employees for change performance, organize productive collaboration, apply reward system, and build employees' accountability i.e. their commitments for the changes in an organization (Bregman, 2016).

Most important aspects of successful change are active management participation, an organizational culture that promotes change, employee involvement, clear and timely communication, suitable organizational structure, availability of change agents, skill teams, properly assigned accountability, adjustment of performance measures, measurement of change success (Hans-Henrik, Oliver \& Neele, 2014; Half, 2015). However, within organizations there are challenges in one or more of these aspects that make change performance difficult. Thereby, many organizational changes fail (Kotter, 2007; Hans-Henrik, Oliver \& Neele, 2014; Sirkin, Keenan \& Jackson, 2015; Kelley, 2016).

The priority challenge that managers encounter is the difficulty in identifying the actual goal of organizational change and the change directions because they haven't full information about the employees' readiness to perform the change. The readiness is represented by interest, opportunity, and ability. The interest supposes professional and material motivation to change performance. The opportunity means compatibility while joint performance of change. The ability is characterized by presence of skills required for the change performance.

Another challenge to successful change management comes in the area of accountability. Personal accountability for change is not adequately leveraged; accountability is not allocated based on employees' readiness to perform change; taking accountability for the process and results of the change is not purposefully stimulated and motivated (Samuel \& Chiche, 2004; Abu-Hussain \& Tilchin, 2019). 
In addition, failure to encourage employees in the change activity prevents their involvement in the change process; the prevailing organization structure limits collaboration among employees; and there is a lack of balance between employee collaboration and competition.

Organizational change can be achieved by change management including change planning and change performance (Neilson, Krings, Aguirre \& Tipping, 2004; Payne, 2005; Hiatt \& Creasey, 2012; Gibbons, 2019). Successful change management can be promoted by a means aimed at overcoming the aforementioned challenges and creating a culture of accountability that intensifies the prospect of organizational change.

The means should direct the change planning accompanied by building employee accountability taken into consideration of assessment of the proposed change and the employees' readiness for change performance. The suggested model is aimed at shaping the change planning process guided by the employees' accountability for organizational change. The model serves as constructive means providing the comprehensive solution for the planning of organizational change through accountability.

\section{Related Research}

The different aspects of relationship between organizational changes and employees' accountability are analyzed in this section.

Melena (2018) created a model for forming work environment favoring employee engagement and improvement of organizational performance. A main element of the model is employee's supportive accountability combining supervision and forceful accountability that induces employee performance. Ayers (2005) affirmed the organizational environment fostering desire and reediness of the employees to take accountability for change should be formed.

Browning (2013) declared a work environment favoring engagement of employees and inducing them to take greater accountability can be created as a result of compliance of certain conditions. These conditions are directed towards providing support of employees from management and team members, the employees' freedom in determination of ways to obtaining results and attaining the goal; required information for making proper decisions; sufficient resources in order to have possibility be accountable; and employees' awareness about roles characterizing subjects and spheres of their accountability for achieving the accountability balance between the process and the results.

Evans, Mike (2017) discovered accountability is catalyzer to accelerated change, sensible employee engagement, strengthened ownership, tireless perseverance, flawless alignment. It encouraged employees, teams, and organizations to successful performance of changes. The author explained how ignite desired accountable behaviors of employees.

Chang, at. al. (2017) examined influence of accountability for process and result of change. The author showed accountability for process enhanced change performance in the short-term, accountability for result induced change performance in the long-term, and accountability for process and results jointly provided flexibility in change performance.

Miller (2006) suggested to consider accountability as a process realization of which is guided by coherent compliance of six principles. The principles are set expectation, invite commitment, measure result, provide feedback, link to consequences, and evaluate effectiveness. The first and second principles are directed towards to induce employees to take accountability for results. The rest principles promote holding the employees' accountability for results.

Bregman (2016) determined five directions to encourage employees' accountability. The directions are clear expectations, clear capability, clear measurement, clear feedback, and clear consequences. The first and second directions contribute to accountability planning.

Samuel \& Chiche (2004) introduced the model which shaped holding personal accountability for problem solving. It is provided by consistent realization of the model components: recognize, own, forgive, self-examine, learn, and take action.

Laughlin (1991) created the organizational change model for clarify change of accountability in organizations. Using this model, Onkutlwile (2002) developed the model for revealing of the factors that influenced on building accountability.

Rashid (2015) defined team mutual accountability causes recognized behavior of team members. The author developed a model setting the relationship between team mutual accountability and team performance through consistent realization of the model components: Team Structures, Team Beliefs, Team Behaviors, and Team 
Outcomes. Grenny (2014) established availability of the relation between team members' mutual accountability and high performance.

Evans Henry (2008) proclaimed improvement of organizational performance is caused by taken into consideration of accountability and construction of relationships among group members on the basis of accountability. Katzenbach \& Smith (2015) declared teams, having the required skills and taking proper commitment and accountability, provided high performance of the organization.

Green, Visser \& Tetlock (2000) claimed joint accounting a reward system and accountability for process and result of change enabled flexibility in change performance.

The analysis of the above publications allows concluding a comprehensive solution for change planning guided by employees' accountability was not made. Such solution should provide: revealing the well-founded directions of the changes; the employees' adjustment to the change directions and their involvement in change process; building reasonable accountability for change; improvement of organizational culture due to properly assigned accountability; formation of teams for change allowing collaboration is not limited by an organization structure; determination of an accountability measures through assessment of change proposals and the employees' readiness to participate in their realization; setting individual measures of accountability for the change proposal realization is resulted by dividing the team accountability measure among team members; the planning of change based on the accountability measures; identifying the most accountable team leader, the most accountability significant change proposal in the change direction, and the most accountability significant direction; and adaptation of compensations for participation in the change planning to the employees' accountability measures.

\section{The three-component model for change planning through accountability}

The suggested model shapes change planning process guided by employees' planned accountability for organizational change through consistent realization of Create, Assess, and Determine components. The model is presented by Figure1.

\section{Create}

- Determine goals and directions of change

- Set requirements to a change proposal content

- Form conditions for qualitative and motivated creation of change proposals

- Familiarize employees with goals, directions, requirements, and conditions

- Create change proposals

- Disseminate change proposals to the public within organization

Assess

- Determine the types of change proposals' assessment

- Establish requirements to assessment of the change proposals

- Form conditions for qualitative assessment of the change proposals and for balancing employee collaboration and competition

- Assess the change proposals

- Assess employee readiness to participate in performance of the change proposals

- Build teams for realization of the change proposals

- Assess the employees' involvement in change process 


\section{Determine}

- Determine measures of employees' accountability for realization of change proposals

- Divide the accountability measure among the team members

- Plan change guided by the accountability measures

- Determine the most accountability significant proposal and the most accountable team leader in the change direction

- Reveal the most accountability significant direction

- Adapt compensation for participation in the change planning to the employees' accountability measures

Figure1. The three-component model for change planning through accountability

\subsection{The Create component}

The purpose of this component is for employees to create the change proposals that accord with the established directions of change, the requirements for content of proposals, and the conditions for creation of proposals.

The requirements are aimed at providing observable, structured, and complete content of change proposal. The conditions are aimed at contributing to qualitative and motivated creation of the change proposals in an organization.

The requirements to content of a proposal are the size of a proposal may be not more than 250 words, and the proposal content must contain the elements: Objective, Description, and Result.

\section{Example1:}

The requirements of the proposal content elements are represented in Table1.

Table1: Requirements of the proposal content elements

\begin{tabular}{|l|l|}
\hline The proposal elements & The requirements \\
\hline Objective & $\begin{array}{l}\text { The objective should be clear, realistic, and achievable within the } \\
\text { determined time period }\end{array}$ \\
\hline Description & $\begin{array}{l}\text { Description should include the proposal concept, conformity of the } \\
\text { proposal to the change direction, novelty and applicability of the } \\
\text { proposal, and the employee's ability to develop the proposal }\end{array}$ \\
\hline Result & $\begin{array}{l}\text { Content should include the possible theoretical or/and applied results } \\
\text { of the proposal performance }, \text { contribution and impact on the needed } \\
\text { change, and anticipated benefits }\end{array}$ \\
\hline
\end{tabular}

The conditions for creation of the change proposals are the proposal must relate to one suitable direction of change, an employee may create only one proposal for the change direction, the number of the proposals that may be created by an employee is restricted, and all participators of the change planning process receive compensation, which is adjusted to their activity.

Employees create the change proposals in conformity with the established change directions and in compliance the aforementioned requirements and conditions, and disseminate their proposals in an organization.

\subsection{The Assess component}

The purpose of this component is to assess the change proposals and employees' readiness to participate in implementation of the peer proposals.

\subsubsection{Assessment of the change proposals}

Assessment of the change proposals is conducted by means of assessment types and conditions that are designed to provide qualitative assessment, balance between collaboration and competition among employees, and involvement of employees in the change process. 
The assessment types that ensure the comprehensiveness of assessment are the peer assessment of the change proposal (or the manager's assessment in the absence of peer assessments), and the assessment of the employees' readiness to participate in realization of the proposal.

The assessment conditions are the employee can be either the proposal-assessor if he or she assesses the proposal, and/or the proposal-participator if he or she is the proposal-assessor who is prepared to participate in the proposal realization; the number of the proposals that an assessor may assess or for which an assessor may be a participator are different and limited, the employee may participate in realization only one proposal from the determined direction of change, the range for a proposal assessment is set from 1 to 10 , the proposal must be assessed with regard to each of the three proposal content elements, and assessment must be in writing.

Example2:

Assessment of the proposal content elements described in Table1 is represented in Table2.

Table2: The example requirements to assessment of the proposal elements

\begin{tabular}{|l|l|l|}
\hline The proposal elements & $\begin{array}{l}\text { The assessment } \\
\text { requirements }\end{array}$ & The written assessment of the proposal element \\
\hline Objective & 2 & $\begin{array}{l}\text { The objective is clear, realistic and achievable } \\
\text { within the required time period. }\end{array}$ \\
\hline Description & 5 & $\begin{array}{l}\text { Aspects of novelty and applicability of the proposal } \\
\text { are forceful. The proposal conforms to the change } \\
\text { direction. The author's ability to develop the } \\
\text { proposal is well founded and clearly described. }\end{array}$ \\
\hline Result & 3 & $\begin{array}{l}\text { The results show contribution, expected benefits, } \\
\text { and impact to the needed change. }\end{array}$ \\
\hline
\end{tabular}

The proposal assessment entails assessment of the proposal elements.

Example3:

Requirements of proposal assessment are presented in Table3.

Table3: The example of requirements for proposal assessment

\begin{tabular}{|l|l|}
\hline $\begin{array}{l}\text { The } \\
\text { requirements } \\
\text { to proposal } \\
\text { assessment }\end{array}$ & Proposal assessment rubrics \\
\hline 1 & $\begin{array}{l}\text { The objective is not clear. Description of the proposal and results absent. } \\
\text { The objective is clear but not achievable within the required time period. The proposal concept } \\
\text { and novelty are disputable. The proposal doesn't conform to the desired change direction. The } \\
\text { author's ability to develop the proposal and the results are not represented. }\end{array}$ \\
\hline 5 & $\begin{array}{l}\text { The objective is clear and realistic but not achievable within the required time period. The } \\
\text { proposal concept is original. The proposal conforms to the change direction. The author's } \\
\text { ability to develop the proposal and applicability of the proposal are not represented. The } \\
\text { results are unconvincing. }\end{array}$ \\
\hline 7 & $\begin{array}{l}\text { The objective is clear, realistic and achievable within the required time period. Aspects of } \\
\text { novelty are forceful. The proposal conforms to the change direction. The author's ability to } \\
\text { develop the proposal is well founded. Results and applicability of the proposal are } \\
\text { unconvincing. }\end{array}$ \\
\hline 10 & $\begin{array}{l}\text { The objective is clear, realistic and achievable within the required time period. Aspects of } \\
\text { novelty and applicability of the proposal are forceful. The proposal conforms to the change } \\
\text { direction. The author's ability to develop the proposal is well founded and clearly described. } \\
\text { The results show contribution, expected benefits, and impact to the needed change. }\end{array}$ \\
\hline
\end{tabular}

Example4:

The proposal assessment of four assessors is presented in Table4. 
Table4: The example of the proposal assessment

\begin{tabular}{|l|l|}
\hline Assessors & Proposal assessment \\
\hline The first & 6 \\
\hline The second & 5 \\
\hline The third & 9 \\
\hline The fourth & 7 \\
\hline
\end{tabular}

The proposal assessments permit determination of the proposal weight, which, in turn, characterizes degree of relevance to the change direction. The proposal weight is determined by summation of the various proposal assessments.

Example5:

The proposal assessments of four assessors are 6, 5, 9, and 7, accordingly (Table4). The proposal weight, therefore, equals 27.

If only the manager has performed the proposal assessment, an alignment coefficient must be introduced to provide a point of comparison of the assessment with assessments of other proposals of the same change direction. The alignment coefficient is fixed as the average of number of assessors of the proposals belonging to the change direction.

Example 6:

The change direction contains three proposals that have been assessed by the employees and one proposal assessed by the manager. The number of the employee-assessors of corresponding proposals are 3,2 , and 4 , accordingly. Then, the alignment coefficient equals 3 .

The manager' assessment of the proposal is equal to 8 . Taking the alignment coefficient (3) into account, the proposal weight equals 24 .

3.2.2 Assessment of employees' readiness to participate in performance of the change proposals

The planned participants of the proposal realization other than the proposal author can be identified by evaluating the assessors' readiness to participate in realization of the change proposal. The evaluation is performed by jointly the proposal-author and the proposal-assessors. If the proposal has been only assessed by the manager, the participants of the proposal realization is choose by jointly the manager and the proposal-author among the assessors of other proposals belonging the same direction.

As previously noted, the readiness includes interest, opportunity, and ability. Interest reflects attention to the proposal, opportunity means compatibility in engaging in joint work, and ability characterizes presence of skills that complement the author's skills to be a productive participant in realization of the change proposal. As a result of evaluating readiness, the planned collaborative team of participants of the proposal realization is formed. The proposal-author as the main participant becomes the team leader.

The weight of planned participation in the change proposal realization can be determined by summation of the individual potential participations' weights. The employees' involvement in realization of the change direction can be determined by summation of the participation weights of the proposals belonging this direction.

In its turn, the weight of individual potential participation is calculated by summation of assessments of the employee's readiness characteristics. The assessment range of the interest and opportunity characteristics is set from 1 to 3 . The assessment range for each skill from ability characteristic is also set from 1 to 3 .

The planned participation weight serves as essential complement to the proposal weight. The total proposal weight is then calculated by adding together the proposal weight and the participation weight.

Example 7:

There are three potential participants in the proposal' realization. The participants' combined skills that complements the author' ability are $\mathrm{s}_{1}, \mathrm{~s}_{2}, \mathrm{~s}_{3}, \mathrm{~s}_{4}, \mathrm{~s}_{5}, \mathrm{~s}_{6}$. The individual skills of first, second, and third participator are $\left(s_{1}, s_{3}\right),\left(s_{2}, s_{4}, s_{5}\right)$ and $s_{6}$, respectively. Assessments of the participants' reediness characteristics and the calculated weights of the individual potential participation in the change proposal realization are presented in Table 5. 
Table5. Assessments of the participants' reediness characteristics

\begin{tabular}{|c|c|c|c|c|c|c|c|c|c|}
\hline \multirow{2}{*}{$\begin{array}{l}\text { The } \\
\text { participators }\end{array}$} & \multirow[t]{2}{*}{ Interest } & \multirow[t]{2}{*}{ Opportunity } & \multicolumn{6}{|c|}{ Ability(the participants' skills) } & \multirow{2}{*}{$\begin{array}{l}\text { The weight } \\
\text { of individual } \\
\text { potential } \\
\text { participation' }\end{array}$} \\
\hline & & & $\mathrm{S}_{1}$ & $\mathrm{~S}_{2}$ & $\mathrm{~S}_{3}$ & $\mathrm{~S}_{4}$ & $\mathrm{~S}_{5}$ & $\mathrm{~S}_{6}$ & \\
\hline $\mathrm{P}_{1}$ & 2 & 2 & 2 & & 3 & & & & 9 \\
\hline $\mathrm{P}_{2}$ & 1 & 2 & & 1 & & 1 & 2 & & 7 \\
\hline $\mathrm{P}_{3}$ & 3 & 1 & & & & & & 2 & 6 \\
\hline
\end{tabular}

In the sample table above, the weight of participation in proposal realization equals 22 . The proposal weight calculated in Example 5 equals 27. Therefore, the total weight of the proposal equals 49 - the sum of the proposal weight and the participation weight.

\subsection{The Determine component}

The purpose of this component is the creation of a change plan including determination of the employees' accountability for the change, the order of realization of change proposals and directions, and the employees' compensation for participation in the change planning.

\subsubsection{Determining the employees' accountability for the change}

Determining the employees' accountability for the proposed change includes determining the measure accountability and dividing of the measure among the team members.

The measure of accountability for the change proposal realization must be correlated with the total weight of the created proposal. This is so because the total weight combines assessment of proposal content by peers and managers, and assessment of the employees' readiness to participate in its realization. Thus, the measure of accountability for the proposal realization can be set equal to the proposal total weight.

The measure of accountability for the proposal realization is the team's measure of mutual accountability. As discussed the above, a team is formed based on assessment of employees' readiness to participate in performance of the change proposal. The team includes the team leader who is the author of the change proposal and a team members who are assessors ready to participate in its realization.

The measure of mutual accountability for the proposal realization must be divided between the team leader and the team members. The measure of accountability of the team leader equals the proposal weight since it was received as a result of the proposal content's assessment. The cumulative accountability measure of the team members equals the planned participation weight since it is a result of assessment of their readiness to participate in the proposal realization. Individual measures of the team members' accountability are determined by dividing the cumulative measure of accountability in accordance with the individual weights of potential participation in the proposal realization.

\section{Example8:}

The total weight of the proposal as calculated in Example 7 was 49 . Hence, 49 serves as the measure of accountability for the proposal realization. The proposal's weight as calculated in Example 5 was 27, which serves as the team leader's accountability measure.

The weight of planned participation in the proposal realization is 22 (Example7). This number represents the cumulative measure of the team members' accountability. This number represents the cumulative measure of the team members' accountability. The weights of potential participation of the first, second, and third team members $-9,7$, and 6 , respectively - represent their individual measures of accountability.

\subsubsection{Planning realization of the proposals from the same change direction}

Planning of realization of the proposals associated with the change direction is performed by their ranking based on the teams' mutual accountability measures. The received order of realization of proposals allows to reveal the most accountable team leader in the change direction who is the author of the proposal with the maximal measure of accountability. The proposal of this author can be represented as the most accountability significant proposal in the change direction. 
If there are multiple proposals with an equally maximal measure of accountability, preference should be given to the proposal with the highest team leader accountability rating, and the author of that proposal should be determined as the most accountable team leader in the change direction.

Example9:

There are four proposals A, B, C, and D associated with the change direction. The measures of mutual accountability for the proposals realization are $46,49,48$, and 49 , respectively. Based on these rating, the planned order of the proposals' realization is $\langle\mathrm{B}, \mathrm{D}, \mathrm{C}, \mathrm{A}\rangle$. The proposals $\mathrm{B}$ and $\mathrm{D}$ have the identical maximal measure of accountability of 49. The authors' accountability measures of the proposals are 27 and 25 , respectively. Accordingly, the author of proposal B is the most accountable team leader in the change direction.

\subsubsection{Planning of the change directions' realization}

Planning of the change directions' realization is performed by their ranking based on the measures of accountability. The measure of accountability for the change direction realization is determined by summation of the measures of accountability for realization of the proposals associated with the direction. The direction with the maximal measure of accountability for its realization is set as the most accountability significant direction.

If there are the change directions with an equally maximal measure of accountability, the combined measures of the team leaders' accountability for realization of the directions are compared. The combined measure of the team leaders' accountability is calculated by summation of the authors' measures of accountability for realization of the proposals associated with the direction. The direction with the highest combined measure is set as the most accountability significant direction. Due to that, reordering of the change directions is performed.

Example10:

Change directions $\mathrm{D}_{1}, \mathrm{D}_{2}$, and $\mathrm{D}_{3}$ contains two, three, and four change proposals, respectively. The accountability measures for realization of the proposals associated with direction $D_{1}$ are 40 , and 45 . The accountability measures for the three proposals associated with direction $\mathrm{D}_{2}$ are 46, 48, and 49. And the accountability measures of the four proposals associated with direction $\mathrm{D}_{3}$ are $32,34,36$, and 41 . Thus, the accountability measures for realization of the directions $\mathrm{D}_{1}, \mathrm{D}_{2}$, and $\mathrm{D}_{3}$ are 85,143 , and 143 , respectively.

The accountability measures for directions $\mathrm{D}_{2}$ and $\mathrm{D}_{3}$ are identical and are higher than $\mathrm{D}_{1}$. Accordingly, to determine the most significant direction, the combined measures of accountability for realization of directions $\mathrm{D}_{2}$ and $\mathrm{D}_{3}$ should be compared. The measures of the authors' accountability for realization of the proposals associated with directions $\mathrm{D}_{2}$ are 24,25 , and 27 , respectively. The measures of the authors' accountability for realization of the proposals associated with directions $\mathrm{D}_{3}$ are $12,15,16$, and 17 , respectively. Therefore, the combined measures of accountability for realization of the directions $\mathrm{D}_{2}$ and $\mathrm{D}_{3}$ are 76 and 60 , respectively. This analysis leads to the conclusion that direction $\mathrm{D}_{2}$ is the most accountability significant direction.

\subsubsection{Determination of the employees' compensations for participation in the change planning}

Employee compensation must be based on the individual's contribution in the change planning. Accordingly, compensation should be adapted to measures of the employees' accountability for change realization.

Employee compensation is determined by consistent performance of the certain procedures.

Initially, the compensation related to the change direction is determined by dividing the amount budgeted for compensation pro rata based upon the measures of accountability for the direction realization.

Example11:

The total compensation budget is $\$ 1484$. There are three change directions $\mathrm{D}_{1}, \mathrm{D}_{2}$, and $\mathrm{D}_{3}$. As seen in Example 10 , the accountability measures for realization of directions $D_{1}, D_{2}$, and $D_{3}$ are 85,143 , and 143, respectively. Consequently, the employees' compensation calculated on the basis of accountability for realization of the change directions is $\$ 340, \$ 572$, and $\$ 572$, respectively.

Next, the employees' compensation related to the change proposals is determined by dividing the compensation for the change directions on a pro rata basis to the measures of accountability for the change proposals realization.

\section{Example12:}

The accountability measures for realization of the change proposals $p_{1}$ and $p_{2}$ associated with direction $D_{1}$ are 40 , and 45, accordingly (Example 10). Employees' compensation for taking accountability for realization of the direction $\mathrm{D}_{1}$ is $\$ 340$. Hence, the employees' compensation for taking accountability for realization of the change proposals $\mathrm{p}_{1}$ and $\mathrm{p}_{2}$ are $\$ 160$, and $\$ 180$, respectively. 
Next, compensations for the author and the participators of the change proposal realization are determined by dividing the employees' compensations for taking accountability for proposal realization according to the accountability measures of the author and participators.

The participants' compensation is divided among the participants pro rata to their individual measures of accountability for participation in the proposal realization.

Example 13:

The employees' compensation for taking accountability for realization of the change proposals $\mathrm{p}_{1}$ is equal to $\$ 160$. The accountability measure for the proposal realization is 40 (Example12). The author' accountability measure is 25 . Therefore, the author' compensation equals $\$ 100$. Respectively, the participants' common compensation is equal to $\$ 60$.

There are two participants in the proposal realization. Their measure of accountability are 6 and 9 , respectively. Consequently, the participants' compensation is $\$ 24$ and $\$ 36$, respectively.

Finally, the employee's total compensation for taking accountability for change can be determined by the total of his or her compensation for authorship of the proposals and the potential participation in realization the certain proposals.

\section{Example14.}

There are three change directions - $\mathrm{D}_{1}, \mathrm{D}_{2}$, and $\mathrm{D}_{3}$. The employee is the author of the proposal associated with direction $\mathrm{D}_{1}$ and a potential participant in realization two proposals from directions $\mathrm{D}_{2}$, and $\mathrm{D}_{3}$. The author's compensation equals $\$ 100$. The employee's compensation for accountability as participant in realization of two proposals is \$26 and \$34. Thus, the employee's total compensation for taking accountability for change is equal to $\$ 160$.

\section{Conclusion}

The suggested three-component model can serve as the constructive tool for the planning of organizational change. The model provides the comprehensive solution for change planning through accountability.

Comprehensiveness of the solution is expressed by building reasonable accountability for change; determining the accountability measures which are derived from assessment of change proposals combined with the employees' readiness to participate in their realization; forming teams for the change proposals realization based on assessment of employees' readiness to participate in the change; assessing the employees' involvement in change process; allocating the measure of mutual accountability for the change proposal realization among team members by quantitative assessment of personal readiness for the change; planning change guided by the accountability measures; identifying the most accountable team leader, the most accountability significant change proposal in the change direction, and the most accountability significant direction; and calculating compensations for participation in the planning of change in accordance with the employees' accountability measures.

Application of the proposed model will confer a number of benefits. Proper determination of employee accountability will enhance employees' commitment to change and leverage improvement in organizational change more generally. Pursuit of continuous change will become part of organizational culture. High likelihood for achieving the change goal will be ensured. Well-founded directions of the changes will identify and the employees will be well adjusted to the change directions. Collaboration among employees will not be limited by an organization structure. Balance between collaboration and competition of the employees will be maintained. The employees' change activity will be encouraged what will motivate their involvement in the change process. Personal accountability as a leverage of changes will be used. Taking accountability for process and result of the change will be purposefully stimulated and motivated.

\section{References}

Abu-Hussain, J., \& Tilchin, O. (2019). The Management of Accountability for Innovation in an Organization. International Journal of Business Administration, 10 (5), 65-72.

Ayers, K. (2005). Creating a responsible workplace, HR Magazine, 50(2), 1-3. http://www.engagementisnotenough.com/pdfs/HR Magazine RBW.pdf

Bregman, P. (2016). The Right Way to Hold People Accountable, Harvard Business Review. 
Browning, H. (2013). Accountability: Taking ownership of your responsibility. Center for Creative Leadership: Pfeiffer, Greensboro.

Chang, W., Atanasov, P., Patil, Sh., Mellers, B. A., Tetlock, Ph. E. (2017). Accountability and adaptive performance under uncertainty: A long-term view, Judgment and Decision Making, 12(6), 610-626.

Evans, Henry (2008). Winning with Accountability: The Secret Language of High-Performing Organizations, Corner Stone Leadership Institute.

Evans, M. (2017). Achieve with Accountability, Ignite, Engagement, Ownership, Perseverance, Alignment \& Change, John Wiley \& Sons, Inc.

Gibbons, P. (2019). The science of organizational change. How leaders set strategy, change behavior, and create an agile culture (Leading change in the digital age), Phronesis Media.

Green, M. C., Visser, P. S., \& Tetlock, P. E. (2000). Coping with accountability cross-pressures: Low-effort evasive tactics and high-effort quests for complex compromises, Personality and Social Psychology Bulletin, 26(11), 1380-1391.

Grenny, J. (2014).The best teams hold themselves accountable, Harvard Business Review.

Half, R. (2015). Time for Change: 5 Basic Tenets of Change Management https://www.roberthalf.com/blog/management-tips/time-for-change-5-basic-tenets-of-changemanagement

Hans-Henrik (H.H.) Jørgensen, O. B., \& Neele, F. (2014). Making change work while the work keeps changing. How Change Architects lead and manage organizational change, IBM Corporation.

Hiatt, J., \& Creasey, T. (2012). Change management. The people side of change, Prosci Learning Center

Katzenbach, J.R., \& Smith, D.K. (2015). The Wisdom of teams: Creating the high-performance organization, Harvard Business Publishing.

Kelley, B. (2016). Charting change: A visual toolkit for making change stick, Palgrave Macmillan, 1st.ed.

Kotter, J. P. (2007). Leading Change: Why Transformation Efforts Fail, Harvard Business Review.

Laughlin, R. (1991). Environmental Disturbances and Organizational Transitions and Transformations: Some Alternative Models. Organization Studies, 209-232.

Melena, S. (2018). Supportive Accountability: How to Inspire People and Improve Performance, Melena Consulting Group.

Miller, B. C. (2006) Keeping Employees Accountable for Results: Quick Tips for Busy Managers, AMACOM; 1 edition.

Neilson, G., Krings, J., Aguirre, D., \& Tipping, A. (2004).Ten guiding principles of change management, Strategy \& Formerly Booz \& Company.

Onkutlwile, O. (2002). Management Accounting, Accountability and Organizational Change: The case of Botswana Firms, Sheffield Hallam University, http://shura.shu.ac.uk/20154/1/10697461.pdf

Payne, V. (2005). Planning and Managing Change, AMA Self-Study.

Rashid, F. (2015). Mutual Accountability and Its Influence on Team Performance. Doctoral dissertation, Harvard University, Graduate School of Arts \& Sciences. https://dash.harvard.edu/bitstream/handle/1/14226095/RASHID-DISSERTATION-2015.pdf?sequence=4

Samuel, M., \& Chiche, S. (2004). The Power of personal accountability: Achieve what matters to you, Xephor Press, New York.

Sirkin, H., Keenan, P., \& Jackson, A. (2015). The hard side of change management, Harvard Business Review 